Short Communication

\title{
Level of agency in sub-clinical checking
}

\author{
S. Belayachi ${ }^{\mathrm{a}, *}$, M. Van der Linden ${ }^{\mathrm{a}, \mathrm{b}}$ \\ ${ }^{a}$ Cognitive Psychopathology Unit, Department of Cognitive Sciences, University of Liège, Boulevard du rectorat 3 (B33), 4000 Liege, Belgium \\ ${ }^{\mathrm{b}}$ Cognitive Psychopathology and Neuropsychology Unit, University of Geneva, Switzerland
}

\section{A R T I C L E I N F O}

\section{Article history:}

Received 15 February 2008

Available online 15 July 2008

\section{Keywords:}

Obsessive-compulsive disorder

Checking

Action representation

Action identification

Level of agency

Dissociation

\section{Introduction}

Obsessive-Compulsive Disorder (OCD) is characterized by both obsessions and compulsions. Obsessions are recurrent unwanted intrusive thoughts or images, that particularly concern worries about contamination (e.g., becoming contaminated by shaking somebody's hands) or repeated doubts (e.g., concerns about having left a door unlocked). Compulsions refer to urges to perform mental or physical acts in a repeated or stereotyped way, of which repetitive checking (e.g., checking locks, lights and appliances) and repetitive washing (e.g., hand washing, house cleaning) are probably the most common (Rasmussen \& Eisen, 1994; Skoog \& Skoog, 1999). Though compulsions are usually conceptualized as aimed to prevent feared harmful situations, it seems that they can also be triggered by discomfort associated to particular sensations of incongruity or failure (i.e., uncomfortable sensations of incorrectness, feelings of "things not being just right", and imperfection; Coles, Frost, Heimberg, \& Rhéaume, 2003). Interestingly, repeated actions in compulsive checking are thought to reflect an inability in achieving a 'sense of task completion' (Szechtman et al., 2001); and recent studies have related checking symptoms to 'not just right' experiences (Coles et al., 2003; Tolin, Brady, \& Hannan, 2008), while washing symptoms may be specifically motivated by harm avoidance (Tolin et al., 2008).Thus, washing individuals may wash repeatedly with the abstract purpose to avoid negative outcomes (e.g., avoiding contamination) or to achieve a particular goal (e.g., purifying the head), whereas people with checking proneness may repeat action until they feel that it has been correctly accomplished (i.e., "just right" feelings), regardless of goal attainment.

Consistently, several studies have suggested that checking behaviors may be specifically related to deficits affecting action processing such as action planning (e.g., van den Heuvel et al., 2005) and action monitoring (e.g., Hajcak \& Simons,

\footnotetext{
* Corresponding author. Fax: +32 43662808.

E-mail address: sanaa.belayachi@ulg.ac.be (S. Belayachi).
} 
2002). More specifically, it has been suggested that the involvement of action planning deficits in checking behaviors is related to a defective use of internal representations to guide the course of action, which may lead checkers to rely on external cues and to repeat an action until they get cues informing them that the action has been completely performed (Purcell, Maruff, Kyrios, \& Pantelis, 1998). Similarly, the postulated overactive action monitoring in checking individuals has been interpreted as a conflict between external outcomes of actions and internal representations, which may lead checkers to detect an inconsistent 'error signal' and to correct actions through checking (Pitman, 1987). From this perspective, checkers' unconventional action regulation mechanisms might stem from their defective representation of goal-directed habitual actions. The goal-directedness of any habitual action causes that action to be mentally represented with strong links between a goal and the instrumental features that serve it, and to be monitored according to its internal related goal (Aarts \& Dijksterhuis, 2000). Such goal representations allow people to understand why they are doing what they are doing in the course of an action and how far they are from a desired end state; on the other hand, performing habitual actions with unavailable or irrelevant goal representations may simply disorganize the action flow (Vallacher \& Wegner, 1989). The present study aimed to further explore the nature of habitual action representation in compulsive checking.

Action representation can be understood in the context of Action Identification Theory (AIT), which proposed a framework for understanding action regulation according to its representation (Vallacher \& Wegner,1985, 1989). This theory proposed that any behavior can be identified within a cognitive hierarchy of meanings, in which the lower-levels represent instrumental features, and the higher-levels relate to the desired goal and outcomes of the action. The particular level at which actions are identified is thought to reflect the accessibility of a particular representation (movements vs. goal). For example, identifying the act of 'locking the door' as 'putting a key in the lock' shows that the instrumental representation is most accessible, whereas identifying this act as 'securing the house' reflects the predominant accessibility of the goal representation. Thus, actions are identified on the basis of a particular level of representation; higher-level action identification is used to perform and monitor well-practiced, routine actions or behaviors, while lower-level identification is used to perform and monitor novel and recently learned actions and behaviors. When an act can be identified at both high and low-levels (as in the case of habitual actions), people adopt higher (more meaningful) levels of action identification over lower-level ones (Vallacher \& Wegner, 1987); however, whenever an act becomes complex or has been disrupted, people tend to adopt lower-levels.

Nevertheless, Vallacher and Wegner (1989) identified individual differences in the way people understand what they are doing according to their predominant level of action identification (i.e., level of personal agency). The tendency to identify action at a uniformly high-level, that is, according to its purpose and implications, refers to a high-level of agency; conversely, the tendency to identify action at a uniformly low-level, that is, based on its procedural aspects and motor subcomponent, refers to a low-level of agency. The authors also associated the level of personal agency with distinct modes of action. Because high-level of agency maybe related to the ability to easily adopt an appropriate level of identification for different types of actions, high-level agents tend to have an overall greater efficiency in everyday actions (e.g., less disruption, fewer resources needed and better goal completion in everyday behaviors). In contrast, chronic low-level of action identification (i.e., low-level of agency) may be related to more difficulties in adapting representations according to action constraints, leading people to have more disruption of the action flow (e.g., emergence of new courses of action, more attention focused on details of performance, doubts about whether the action has been completed; Vallacher \& Wegner, 1989).

Furthermore, Vallacher and Wegner emphasized that the way people understand what they are doing contributes to the way they understand themselves. Indeed, high-level identities allow one to extract intentions behind actions, which can provide meaningful depictions of the self. Conversely, low-level identities have little significance for self-understanding, what can make low-level agents uncertain about what they are really like. Thus, the level of personal agency may be related to some aspects of the sense of self, such that a high-level of agency allows people to extract abstract self-knowledge from actions and thus may give them a coherent and stable understanding of themselves. A low-level of agency, on the other hand, prevents people from doing this and may therefore have a less strong and coherent sense of self.

In sum, the level of agency refers to the preferential level at which actions are generally identified, which reflects the internal representation (goal vs. movements) that is generally activated during an action, and promotes distinct modes of action. One factor that is directly relevant to the present study is the potential for action deregulation of low-level of agency (i.e., predominant identification and accessibility of instrumental features). Indeed, in the absence of goal representations, gestural representations guide actions (Wegner, Vallacher, Macomber, Wood, \& Arps, 1984) and chronic low-level identification is thought to promote 'signals of inconsistency and error', particularly during routine actions (Vallacher \& Wegner, 1989). Consistently, compulsive checking has been specifically related to abnormal action regulation that may lead individuals to repeat their actions (e.g., overactive action monitoring; Hajcak \& Simons, 2002). Furthermore, it has been proposed that doubts about the performance and repetition of action, which characterize checking, maybe related to the focus of 'attention to low-level gestural units of behavior rather than to goal-related higher-level units that are normally used in action flow parsing' (Boyer \& Liénard, 2006, p. 1).

In light of this model, we could postulate that checking individuals may identify their habitual actions at a preferential low-level. Conversely, washing patients have been reported to identify habitual action at a high-level as compared to nonOCD controls (Dar \& Katz, 2005). In their study, Dar and Katz used an item related to washing symptoms, i.e., the habitual act of washing hands, associated with 22 identities varying in level of abstraction (11 identities representing low-level identification such as 'I run water over my hands' and 11 identities representing high-level identification such as 'I feel cleansed'), for which patients and non-OCD controls had to indicate their degree of agreement with each identity. Their results 
suggested that patients may conduct their rituals with an abstract purpose in mind (e.g., 'I clean myself internally'), rather than according to concrete and mechanistic details. In line with the recent suggestion according to which washing symptoms may be specifically motivated by the goal of avoiding harm (Tolin et al., 2008), while checking symptoms may be particularly related to "not just right" experiences and concerns with the details of action (Coles et al., 2003; Tolin et al., 2008), our main purpose in this study was to confirm that checking symptoms are specifically associated with action identification preferentially at a low-level (i.e., low-level of agency), as compared to washing symptoms.

We will also examine the relationship between low-level of agency and altered sense of self in checking. Numerous studies have observed specific links between checking symptoms and dissociative states (e.g., Rufer, Fricke, Held, Cremer, \& Hand, 2006). Thus, we will also examine whether proneness to dissociation in checking symptoms maybe related to low-level of agency.

Those issues will be examined in sub-clinical checkers, who have been found to have clinical features and cognitive impairments similar to those identified in checking patients (e.g., Muris, Merckelbach, \& Clavan, 1997; Zermatten, Van der Linden, Larøi, \& Ceschi 2006b). This approach has the potential benefit of overcoming some of the methodological problems linked to the use of clinical samples (e.g., pharmacological and psychological therapies that may interact with dependent measures).

\section{Method}

\subsection{Participants}

One hundred and forty-seven undergraduates from the University of Liège took part in the study. The subjects were tested in group sessions (four subjects maximum). Data from 24 participants had to be discarded as values were missing in their questionnaires. Homogeneity statistic tests ${ }^{1}$ revealed that the mean scores for all the measures did not differ from those of the remaining participants ( $p s>.53$ ).The reported results are from the remaining 123 participants (55 males and 68 females). Their mean age was 21.7 years $(S D=2.9$ years).

\subsection{Materials and procedure}

Informed consent was obtained from all participants following a full explanation of the experimental procedure. Detailed written and oral instructions explained that participants would be asked questions about different aspects of routine actions. They were participating anonymously and on a volunteer basis. Three different questionnaires were administered to investigate the level of agency, the level of checking symptoms and the proneness to dissociation, respectively. These measures were counterbalanced across subjects.

Behaviour Identification Form (BIF, Vallacher \& Wegner, 1989). The BIF is an instrument designed to measure individual differences in action identification level across an array of routine actions (i.e., level of personal agency). Each item of the BIF consists of an action followed by two alternatives or 'identities', one of which is lower and one higher in level. For each action, participants had to choose the alternative that best describes the action they would carry out. The number of highlevel identities chosen defined the subjects' level of agency. The French version of the BIF has been validated in a previous study intended to replicate the Vallacher and Wegner (1989) study of level of agency (Belayachi \& Van der Linden, 2008).

In our replication study, a 35-item version was first created; the items were derived from the original version translated into French, and the authors also generated some new items. In order to ensure that identities were unambiguous, 20 judges familiar with AIT were asked to rate the level of identification of each alternative identity. Since there were no ambiguous identities, this 35-item form of the BIF was administered to a sample of 246 undergraduates (136 women and 110 men). Analysis showed good internal consistency (Cronbach's $\alpha=89$ ); and the item-total correlations for these items ranged from -.46 to .78 with a mean of .43 . Using a procedure similar to that adopted by Vallacher and Wegner, we used the mean itemtotal correlation of .43 as the criterion for item inclusion, which reduced the instrument to 23 items. The 23 remaining items constituted the final French version of the BIF, which had an internal consistency of .90. In addition, an exploratory factor analysis (principal-axis solution) revealed the existence of one factor, suggesting that the French version of the BIF is indeed a one-dimensional scale.

Obsessive-Compulsive Inventory-Revised (OCI-R, Foa et al., 2002). The validated French version of the OCI-R (Zermatten, Van der Linden, Jermann, \& Ceschi, 2006a) is a self-report questionnaire that consists of 18 items evaluating OCD symptoms and is composed of six subscales (each containing three items): 'Washing', 'Obsessing', 'Hoarding', 'Ordering', 'Checking' and 'Neutralizing'. The checking subscale consists of the following three items: "I check things more often than necessary", "I repeatedly check doors, windows, drawers, etc.", "I repeatedly check gas and water taps and light switches after turning them off"; and the washing subscale includes these three items: "I find it difficult to touch an object when I know it has been touched by strangers or certain people", "I sometimes have to wash or clean myself simply because I feel contaminated", "I wash my hands more often and longer than necessary". Participants were asked to determine to what extent the situation

\footnotetext{
1 The homogeneity statistic test assessed the confidence interval for the difference between two proportions. The procedure for obtaining such an interval is based on the sample proportions, p1 and p2, from their respective overall populations (Easton \& McColl, 1997).
} 
Table 1

Mean scores and SDs for level of agency, dissociation and OCD measures

\begin{tabular}{ll}
\hline Dependent variables & Score $(S D)$ \\
\hline Level of agency & $13.7(4.2)$ \\
Dissociation & $30.3(20.2)$ \\
DES absorption & $18.4(11.9)$ \\
DES depersonalization & $12.5(12.4)$ \\
DES dissociative amnesia & $20.4(13.8)$ \\
DES total score & $3.1(2.6)$ \\
OCD & $1.7(2.3)$ \\
OCI-R checking & $17.9(10.2)$ \\
OCI-R washing & \\
OCI-R total score & \\
\hline
\end{tabular}

Note: $S D$, standard deviation.

described in each particular statement had distressed them during the past month, using a 5-point scale ( 0 = 'not at all'; 4 = 'extremely'). Total scores range from 0 to 72 ; the OCI-R checking and washing subscales scores both range from 0 to 12. This French version of the OCI-R has good overall psychometric properties and a factorial structure that is identical to that observed in Foa et al.'s (2002) original English version (Zermatten et al., 2006a).

Dissociative Experience Scale-II (DES, Carlson \& Putnam, 1993). The validated French version of the DES (Darves-Bornoz, Degiovanni, \& Gaillard, 1999) is a 28-item self-report instrument in which subjects are asked to answer questions by marking a horizontal line (with $0 \%$ of the time on the left and $100 \%$ of the time on the right) to show how often the dissociative events happen. Three forms of dissociation can be measured with the DES: 'Absorption' (i.e., narrowing or expanding the focus of attention), 'Depersonalization/Derealization' (i.e., respectively, a loss of sense of self and a loss of sense of reality) and 'Dissociative amnesia' (i.e., loss of autobiographical memory). Mean scores for the three subscales and the entire scale range from 0 to 100. This French version of the DES has good overall psychometric properties and a factorial structure that is similar to that observed in Carlson and Putnam (1993) original English version (Darves-Bornoz et al., 1999).

\section{Results}

Cronbach's alphas indicated good internal consistency for the level of agency (BIF: .89), OCD measures (OCI-R total score: .85; checking: .76; washing: .75), ${ }^{2}$ and dissociation measures (DES total score: .93; absorption: .83; depersonalization: .81; dissociative amnesia: 84 ). The sample means scores for all these measures are shown in Table 1.

A homogeneity statistic test confirmed that the mean score on the BIF obtained in this study $(M=13.7 ; S D=4.2)$ did not differ from that observed in a sample of 246 undergraduates in the Belayachi and Van der Linden (2008) study ( $p>.5$ ); it was also similar to that in a heterogeneous sample of 1404 participants (undergraduates and staff faculty, gynaecological outpatients, juvenile detainees) in Vallacher and Wegner's study $(p>.2)$. The overall DES mean score observed in this study $(M=20.4 ; S D=13.8)$ has been shown to be comparable, through homogeneity statistic tests, to DES means that have been observed in an American sample of 308 college students in the Zingrone and Alvarado's (2002) study ( $p>.5$ ); as in a French sample of 248 undergraduates in Moyano and Claudon's (2003) study $(p>.4)$. In addition, DES subscales scores are in agreement with other studies in which absorption dissociation form $(M=30.3 ; S D=20.2)$ is most frequently observed in the general population (e.g., Ross, Joshi, \& Currie, 1991). Mean scores for OCD dimensions showed that checking symptoms ( $M=3.1$; $S D=2.6)$ are more frequent than washing symptoms $(M=1.7 ; S D=2.3)$, replicating previous studies in which checking was found to be more frequent in the general population than washing (e.g., Zermatten \& Van der Linden, in press). Nevertheless, homogeneity statistic tests revealed that checking and washing mean scores were significantly higher in our sample than in a French-speaking sample of 419 undergraduates in the Zermatten and Van der Linden's (in press) study ( $p s<.02)$; whereas OCI-R total mean score was comparable across the two studies $(p>.3)$.

Pearson correlations were performed in order to explore associations between the measures of OCD, the level of agency and dissociation. These analyses revealed that the level of agency was negatively correlated with the checking subscale of the OCI-R $(r=-.18 ; p<.05)$, but not with the washing subscale $(r=-.03 ; p>.7)$. A correlation analysis conducted between the dissociation and OCD measures revealed that they were significantly correlated, as shown in Table 2 . However, correlations between the measures of the level of agency and dissociation failed to reach significance $(p>.4)$.

In order to explore the independent contribution of checking symptoms on the level of agency, with the effect of the washing variable removed, we performed a multiple regression analysis on the level of agency, using the OCI-R checking and washing subscale scores as independent variables. The results indicated that checking was a significant predictor of the level of agency $(t=-2.01, p<.05, \beta=-.20)$, whereas washing was not related to level of agency $(t=0.5, p<.7, \beta=.04)$.

\footnotetext{
${ }^{2}$ As only the checking and washing subscales of the OCI-R were used, we report the coefficients for these subscales only.
} 
Table 2

Pearson correlations between checking and washing subscale scores and level of agency and DES scores

\begin{tabular}{lll}
\hline & OCD dimensions \\
\cline { 2 - 3 } & Checking \\
\hline Level of agency &.$- \mathbf{1 8}^{*}$ \\
Dissociation & \\
Absorption &. .03 \\
Depersonalization & $.7^{* *}$ \\
Dissociative amnesia & $\mathbf{. 3 2}^{* *}$ \\
DES total & $\mathbf{. 3 1}^{* *}$ \\
\hline
\end{tabular}

${ }^{*} p<.05$.

$p<.01$.

In order to investigate the specific relationship between dissociation and checking, as compared to washing, we performed another multiple regression analysis on the three dissociative measures, using the OCI-R checking and washing subscale scores as independent variables. These results indicated that checking was significantly and specifically related to absorption $(t=2.14, p<.04, \beta=.20)$, depersonalization $(t=3.03, p<.01, \beta=.28)$, and dissociative amnesia $(t=2.95, p<.01$, $\beta=.27)$, while washing showed no independent relationship with dissociation $(p s<.06)$.

We did not investigate the contribution of level of agency to checkers' proneness to dissociation because level of agency and dissociation were not correlated.

\section{Discussion}

The aim of this study was to examine whether checkers identify various habitual actions at a uniformly lower-level rather than according to the related goals. The results showed that checking symptoms are specifically related to the tendency to identify routine actions in terms of concrete mechanistic details. These results are consistent with the recent theoretical suggestion that ritualized action is not connected to a representation of a goal, which is not in fact accessible (Boyer \& Liénard, 2006). In the absence of goal representations, gestural representations guide actions (Wegner et al., 1984), which may lead checking-prone individuals to be 'task-oriented' (i.e., focused on performance), rather than 'goal oriented' (i.e., focused on implications). In other words, checkers seem to act without having any representation that allows them to understand why they are executing certain actions. This may explain the repeated enactment of routine actions regardless of the obvious achievement of the goal.

In addition, acting with such low-level representations in mind is thought to promote 'signals of inconsistency and error', particularly during routine actions (Vallacher \& Wegner, 1989). Numerous studies have observed the presence of inappropriate error-processing signals in checking individuals that may lead them to repeat their actions (e.g., overactive action monitoring; Hajcak \& Simons, 2002). Vallacher and Wegner (1989) proposed that people with low-levels of agency are more likely to adopt alternative higher-level meanings from external cues, leading them to orient the course of action according to the new external alternative identity by establishing a new course of action. In the AIT framework, checking individuals might be characterized by preferential access to instrumental representations, rather than goal representations. This could prompt them to control their actions according to situational cues, leading to the emergence of successive alternative meanings that necessitate updating the current action, which may then engage them in new courses of action. This external control of action is thought to promote impulsive behaviors so that low-level agents may be more likely to respond automatically to situational pressures, regardless of the ongoing activity (Vallacher \& Wegner, 1989). Interestingly, OCD symptoms have been recently related to different facets of impulsivity (Zermatten \& Van der Linden, 2008). However, what remains unclear is the nature of the psychological mechanisms specifically involved in the relationship between impulsivity and OCD symptoms.

Another important outcome of this study is the fact that a low-level of agency is specifically related to checking, and not to washing. It should be noted that Dar and Katz (2005) recently showed that washing symptoms were associated with a highlevel of action identification, an association that is not observed in our study. However, Dar and Katz examined the level of action identification in washers by using an action specifically related to their obsessions and compulsions, namely the act of washing their hands, whereas this was not the case in our study. In this vein, it would also be important to explore the level at which actions related to checking symptoms (e.g., turning off the stove) are identified by checking individuals. Nonetheless, our results are in line with previous studies in which checking and washing symptoms had different patterns of cognitive impairments (for a review, see Muller \& Roberts, 2005); supporting the idea that 'OCD might have to be conceptualized as a spectrum of multiple, potentially overlapping syndromes rather than a unitary nosologic entity' (Mataix-Cols et al., 2004, p. 1).

A secondary investigation in this study explored the links between the low-level of agency and altered sense of self in dissociative states in compulsive checking. The results showed that checking symptoms were specifically associated with the tendency to manifest an altered sense of self, whereas no independent relationship was found between dissociation 
and washing symptoms; what replicates previous results obtained in a clinical sample (Rufer et al., 2006). However, there was no correlation between the level of agency and dissociation, which contradicts our prediction that the presence of dissociative states in compulsive checking may be related to low-level of agency. We also failed to replicate previously observed links between level of agency and sense of self (Vallacher \& Wegner, 1989), though the absence of an association between agency levels and dissociation in our study may stem from the different dimension of the self that we assessed. Indeed, Vallacher and Wegner related level of agency to a conceptual dimension of the self such as self-definition through personality traits, which constitutes knowledge about the self that is not available to immediate perceptual experience (Morin \& Michaud, 2007). In our study, on the other hand, we related level of agency to a phenomenal dimension of the self such as one's direct experience of oneself or the world. Overall, this suggests that action identification levels may be specifically related to abstract knowledge of the conceptual self, rather than to immediate perceptual experience of the self. Future studies should explore the connection between low-level of agency and altered sense of self in checking individuals by using measures that assess the conceptual dimension of the self.

Before concluding, we should emphasize that the correlational method we used prevented us from determining the nature of the relationship between low-level of agency and compulsive checking. Indeed, what remains unclear is whether lowlevel of agency leads people to check their actions, or whether checking proneness may provoke a chronic low-level of action identification. It is possible that a lack of goal accessibility during actions may lead people to misunderstand what they have just done and consequently to check their actions. Conversely, individuals with checking proneness may identify routine actions at a preferential low-level in order to make sure that their routine actions have been performed successfully. One way of answering this question would be to explore the extent to which inducing low-level of action identification may triggers doubts about self-performed actions and checking behaviors, and the extent to which inducing checking may engender spontaneous low-level of action identification. Nevertheless, we assume that low-level of agency may only constitute one of the numerous factors implicated in checking, such as anxiety, attentional focus, reality monitoring difficulties and poor memory for actions (Boyer \& Liénard, 2006; Zermatten \& Van der Linden, in press). For example, high-levels of anxiety have been reported to lead individuals to focus attention on details, favoring local perceptual information processing (e.g., Boyer \& Liénard, 2006; Derryberry \& Reed, 1998). Further studies should be conducted on compulsive checking in order to clarify the relationships between low-level of action identification, anxiety and low-level attentional focus. Similarly, the relationship between low-level of identification, difficulties in action memory and reality monitoring should be explored. In this prospect, Vallacher and Wegner (1989) showed that people who identify their routine actions at a preferential low-level are more prone to everyday memory failure and particularly to an inability to remember whether a previous intended action has been executed (e.g., forgetting whether the light has been turned off or whether the door has been locked,...). Accordingly, chronic low-level of action identification maybe responsible for memory failure, which in turn may trigger doubts about action performance and checking behaviors.

In conclusion, this study mainly highlighted the predominantly instrumental identification of actions that may characterize checkers. The results support the idea that checking individuals perform actions that are disconnected from the goal they wish to achieve. This may explain why they have concerns about performance and doubts about the correctness of their actions, and why they repeat them, even after achieving their goal. Although comparably defective action processing has been observed in both sub-clinical and clinical checkers (Ecker \& Engelkamp, 1995; Gehring, Himle, \& Nisenson, 2000; Hajcak \& Simons, 2002; Zermatten et al., 2006b), the impact of chronic checking symptoms on action identification levels remains to be explored. Thus, future studies should examine this issue in individuals with more severe obsessive-compulsive symptoms (i.e., patients with compulsive checking). Further work is also needed to see, on the one hand whether a low-level of agency could be related to checkers' cognitive deficits (e.g., overactive action monitoring) and, on the other hand, to what extent our results can be replicated with checking-related actions (e.g., turning off the stove, locking the door).

\section{Acknowledgments}

This study was supported by a grant from the French-speaking community of Belgium (Action Recherche Concertée, Convention 06/11-340).

\section{References}

Aarts, H., \& Dijksterhuis, A. (2000). Habits as knowledge structures: Automaticity in goal-directed behavior. Journal of Personality and Social Psychology, 78, 53-63.

Belayachi, S., \& Van der Linden, M. (2008). A French adaptation of the Behaviour Identification Form, in preparation.

Boyer, P., \& Liénard, P. (2006). Why ritualized behavior? Precaution systems and action parsing in developmental, pathological and cultural rituals. Behavioral and Brain Sciences, 29, 1-56.

Carlson, E., \& Putnam, F. (1993). An update on the Dissociative Experiences Scale. Dissociation, 6, 16-27.

Coles, M. E., Frost, R. O., Heimberg, R. G., \& Rhéaume, J. (2003). "Not just right experiences": Perfectionism, obsessive-compulsive features and general psychopathology. Behaviour Research and Therapy, 41, 681-700.

Dar, R., \& Katz, H. (2005). Action identification in obsessive-compulsive washers. Cognitive Therapy and Research, 29, 333-341.

Darves-Bornoz, J. M., Degiovanni, A., \& Gaillard, P. (1999). Validation of a French version of the Dissociative Experiences Scale in a rape-victim population. Canadian Journal of Psychiatry, 44, 271-275.

Derryberry, D., \& Reed, M. A. (1998). Anxiety and attentional focusing: trait, state and hemispheric influences. Personality and Individual Differences, 25, 745-761. 
Easton, V. J., \& McColl, J. H. (1997). Statistics Glossary. [Online]. Available from http://www.stats.gla.ac.uk/steps/glossary/hypothesis_testing.html\#pvalue (10 January 2004).

Ecker, W., \& Engelkamp, J. (1995). Memory for actions in obsessive-compulsive disorder. Behavioural and Cognitive Psychotherapy, 23, 349-371.

Gehring, W. J., Himle, J., \& Nisenson, L. G. (2000). Action monitoring dysfunction in obsessive-compulsive disorder. Psychological Science, 11, 1-6.

Foa, E. B., Huppert, J. D., Leiberg, S., Langner, R., Kichic, R., Hajcak, G., et al (2002). The Obsessive-compulsive inventory: Development and validation of a short version. Psychological Assessment, 14, 485-496.

Hajcak, G., \& Simons, R. F. (2002). Error-related brain activity in obsessive-compulsive undergraduates. Psychiatry Research, $110,63-72$.

Mataix-Cols, D., Wooderson, S., Lawrence, N., Brammer, M. J., Speckens, A., \& Phillips, M. L. (2004). Distinct neural correlates of washing, checking, and hoarding symptom dimensions in obsessive-compulsive disorder. Archives of General Psychiatry, 61, 564-576.

Morin, A., \& Michaud, J. (2007). Self-awareness and the left inferior frontal gyrus: Inner speech use during self-related processing. Brain Research Bulletin, 6, 387-396.

Moyano, O., \& Claudon, P. (2003). Stern dissociative experiences in a French post-graduate students group: On depersonalization. Annales MédicoPsychologiques, Revue Psychiatrique, 161, 183-189.

Muller, J., \& Roberts, J. E. (2005). Memory and attention in obsessive-compulsive disorder: A review. Journal of Anxiety Disorders, 19, 1-28.

Muris, P., Merckelbach, H., \& Clavan, M. (1997). Abnormal and normal compulsions. Behaviour Research and Therapy, 35, $249-252$.

Pitman, R. K. (1987). A cybernetic model of obsessive-compulsive pathology. Comprehensive Psychiatry, 28, 334-343.

Purcell, R., Maruff, P., Kyrios, M., \& Pantelis, C. (1998). Cognitive deficits in obsessive-compulsive disorder on tests of frontal-striatal function. Biological Psychiatry, 43, 348-357.

Rasmussen, S., \& Eisen, J. (1994). The epidemiology and clinical features of obsessive-compulsive disorder. Psychiatric Clinics of North America, 15, 743-758.

Ross, C. A., Joshi, S., \& Currie, R. (1991). Dissociative experiences in the general population: A factor analysis. Hospital \& Community Psychiatry, 42, $297-301$.

Rufer, M., Fricke, S., Held, D., Cremer, J., \& Hand, I. (2006). Dissociation and symptom dimensions of obsessive-compulsive disorder: A replication study. European archives of psychiatry and clinical neuroscience, 256, 146-150.

Skoog, G., \& Skoog, I. (1999). A 40 year follow-up of patients with obsessive-compulsive disorder. Archive of General Psychiatry, $56,121-127$.

Szechtman, H., Eckert, M. J., Tse, W. S., Boersma, J. T., Bonura, C. A., McClelland, J. Z., Culver, K. E., \& Eilam, D. (2001). Compulsive checking behavior of quinpirole-sensitized rats as an animal model of obsessive-compulsive disorder (OCD): Form and control. BMC Neuroscience, 2, 4-18.

Tolin, D. F., Brady, R. E., \& Hannan, S. (2008). Obsessional beliefs and symptoms of obsessive-compulsive disorder in a clinical sample. Journal of Psychopathology and Behavioral Assessment, 30, 31-42.

Vallacher, R. R., \& Wegner, D. M. (1985). A theory of action identification. Hillsdale, NJ: Lawrence Erlbaum Associates.

Vallacher, R. R., \& Wegner, D. M. (1987). What do people think they're doing? Action identification and human behavior. Psychological Review, 94, 3-15.

Vallacher, R. R., \& Wegner, D. M. (1989). Levels of personal agency: Individual variation in action identification. Journal of Personality and Social Psychology, 57, 660-671.

van den Heuvel, O. A., Veltman, D. J., Groenewegen, H. J., Cath, D. C., van Balkom, A. J., van Hartskamp, J., et al (2005). Frontal-striatal dysfunction during planning in obsessive-compulsive disorder. Archives of General Psychiatry, 62, 301-309.

Wegner, D. M., Vallacher, R. R., Macomber, G., Wood, R., \& Arps, K. (1984). The emergence of action. Journal of Personality and Social Psychology, 46, 269-279.

Zermatten, A., \& Van der Linden, M. (in press). Phenomenal characteristics of memories of daily actions in checking-prone individuals. Applied Cognitive Psychology.

Zermatten, A., \& Van der Linden, M. (2008). Impulsivity in non-clinical persons with obsessive-compulsive symptoms. Personality and Individual Differences, $44,1824-1830$.

Zermatten, A., Van der Linden, M., Jermann, F., \& Ceschi, G. (2006a). Validation of a French version of the Obsessive-Compulsive Inventory-Revised in a nonclinical sample. European Review of Applied Psychology, 56, 151-208.

Zermatten, A., Van der Linden, M., Larøi, F., \& Ceschi, G. (2006b). Reality monitoring and motor memory in checking-prone individuals. Journal of Anxiety Disorders, 20, 580-596.

Zingrone, N. L., \& Alvarado, C. S. (2002). The Dissociative Experiences Scale-II: Descriptive statistics, factor analysis, and frequency of experiences. Imagination, Cognition and Personality, 21, 145-157. 